\title{
OPOSSum - An Online Portal to Collect and Share Semantic Service Descriptions
}

\author{
Ulrich Küster, Birgitta König-Ries, and Andreas Krug \\ Institute for Informatics, Friedrich-Schiller-University Jena, 07743 Jena, Germany \\ ukuester | andreas .krug | koenig@informatik. uni-jena.de
}

\section{Introduction and Motivation}

Despite of a wealth of theoretical work in the area of semantic web services (SWS), surprisingly little effort has been spent towards the comparative evaluation of the competing approaches [1]. This lack of experimentation is a critical blocker to the further development of the field as well as the industrial adoption of the developed technologies. According to recent studies [2], the main problem in evaluating SWS technology is the lack of sufficient test data, i.e. collections of semantically annotated web services. The creation of such collections requires community effort, if they are to be sufficiently large and diverse to allow for meaningful evaluation. This is only possible, if tools exist, which support to collect, share, and improve SWS descriptions collaboratively. So far, such tools have been lacking. The recently set up SWS-TC-Wiki ${ }^{1}$ is a first attempt in this direction. However, while it enables easy sharing of semantic descriptions, it does not allow to edit existing descriptions easily or to search for descriptions with particular properties. It is the goal of OPOSSum, the Online Portal for Semantic Services, presented in this poster to improve the situation by making it easy to collect, search for, and compare semantic service descriptions across formalisms.

\section{Design and Implementation}

The design of OPOSSum has been motivated by three principles: First, it must become easier to share, reuse and improve existing data. Second, the structure, documentation and usability of the current test collections must be improved. Third, unlike current collections, which are all specific to a particular formalism, future test beds should allow the comparison and usage of SWS descriptions across formalisms.

As a result of these considerations and unlike all existing collections, OPOSSum is structured around the notion of a service, independent from a particular service description. A service in OPOSSum is primarily described by a natural language text. To add more structure and support more precise searching, a service's parameters (inputs and outputs) should be declared explicitly and described in natural language in addition to the general description of the service. To add more semantics without binding to a particular formalism, parameter

\footnotetext{
${ }^{1}$ http://www-ags.dfki.uni-sb.de/swstc-wiki/index.php/Main_Page
} 


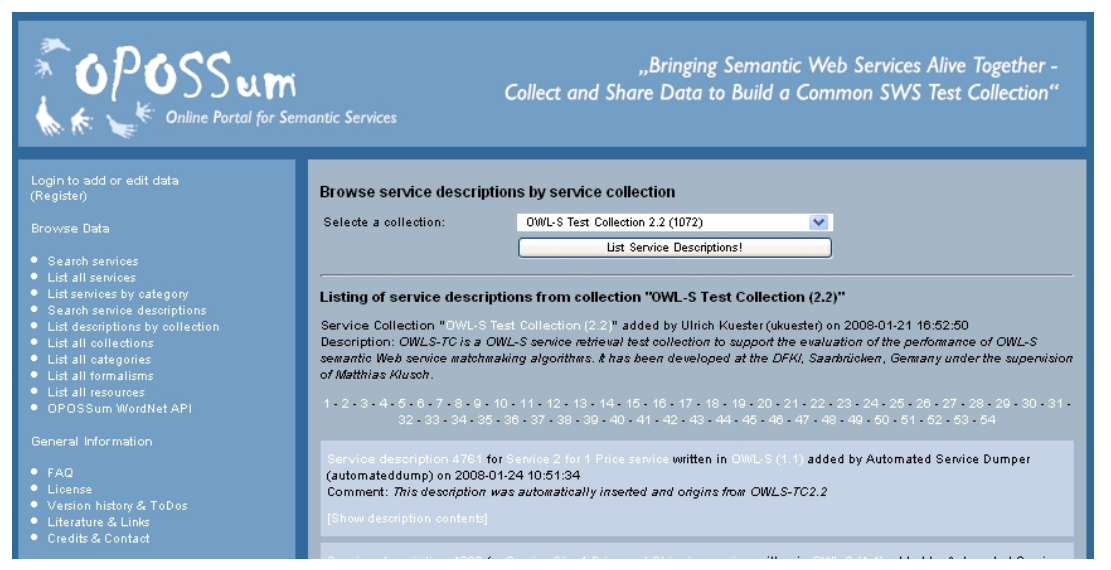

Fig. 1. Screenshot of OPOSSum

types are mapped to WordNet synsets ${ }^{2}$, thus providing a kind of semantics that ensures an excellent compromise between being unambiguous, flexible, easily usable and language/formalism independent. Service descriptions written in any formalism (WSDL, OWL-S, SAWSDL, WSML, ... ) are collected by attaching them to the service (request or offer), they describe. Resources like ontologies or schemas can be added to the system as an independent entity. Descriptions may then refer to the resources that they import. This allows to compute the necessary resources for a given set of service descriptions and thus to automatically assemble test collections with all necessary resources on the fly.

OPOSSum has been implemented as a PHP-based web application and is available online ${ }^{3}$. The existing test collections SWS-TC 1.1 and OWLS-TC 2.2 as well as some services from other sources have been fully integrated. OPOSSum currently contains more than 1300 service descriptions for more than 1250 services, making it the largest publicly available collection of SWS.

\section{References}

1. Küster, U., Lausen, H., König-Ries, B.: Evaluation of semantic service discovery - a survey and directions for future research. In: Postproceedings of the 2nd Workshop on Emerging Web Services Technology (WEWST07) at the 5th IEEE European Conference on Web Services (ECOWS07), Halle (Saale), Germany (2007)

2. Klusch, M., Xing, Z.: Semantic web services in the web: A preliminary reality check. In: Proceedings of the First International Joint Workshop $\mathrm{SMR}^{2}$ on Service Matchmaking and Resource Retrieval in the Semantic Web at the 6th International Semantic Web Conference (ISWC07), Busan, South Korea (2007)

\footnotetext{
${ }^{2}$ WordNet (http://wordnet.princeton.edu/) is a semantic lexicon for the English language developed at Princeton University. It uses the notion of synsets to collect synonyms and disambiguates homonyms.

${ }^{3}$ http://hnsp.inf-bb.uni-jena.de/Opossum/
} 